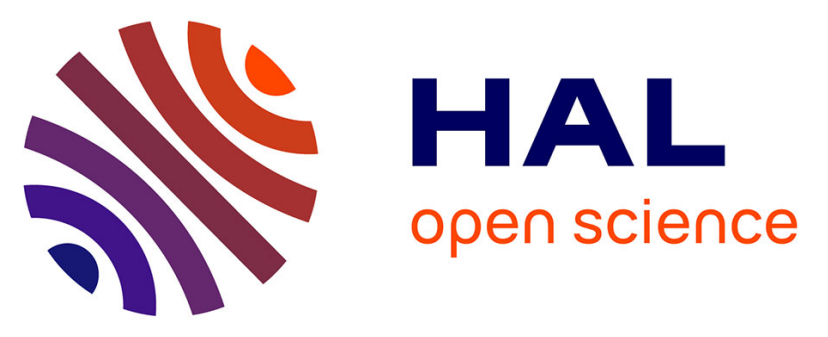

\title{
Three-dimensional time-resolved fluorescence imaging by multifocal multiphoton microscopy for a photosensitizer study in living cells
}

Ariane Deniset-Besseau, Sandrine Leveque-Fort, M.P. Fontaine-Aupart, Gérard Roger, Patrick Georges

\section{To cite this version:}

Ariane Deniset-Besseau, Sandrine Leveque-Fort, M.P. Fontaine-Aupart, Gérard Roger, Patrick Georges. Three-dimensional time-resolved fluorescence imaging by multifocal multiphoton microscopy for a photosensitizer study in living cells. Applied optics, 2007, 46 (33), pp.8045-8051. hal-00691865

\section{HAL Id: hal-00691865 \\ https://hal-iogs.archives-ouvertes.fr/hal-00691865}

Submitted on 27 Apr 2012

HAL is a multi-disciplinary open access archive for the deposit and dissemination of scientific research documents, whether they are published or not. The documents may come from teaching and research institutions in France or abroad, or from public or private research centers.
L'archive ouverte pluridisciplinaire HAL, est destinée au dépôt et à la diffusion de documents scientifiques de niveau recherche, publiés ou non, émanant des établissements d'enseignement et de recherche français ou étrangers, des laboratoires publics ou privés. 


\title{
Three-dimensional time-resolved fluorescence imaging by multifocal multiphoton microscopy for a photosensitizer study in living cells
}

\author{
A. Deniset-Besseau, ${ }^{1,3}$ S. Lévêque-Fort, ${ }^{1,3, \star}$ M. P. Fontaine-Aupart, ${ }^{1,3}$ G. Roger, ${ }^{2,3}$ \\ and P. Georges ${ }^{2,3}$ \\ ${ }^{1}$ Laboratoire de Photophysique Moléculaire, CNRS Unité Propre de Recherche 3361, Univ Paris-Sud, Batiment 210, \\ F-91405 Orsay, France \\ 2Laboratoire Charles Fabry de l'Institut d'Optique, CNRS, Univ Paris-Sud, Campus Polytechnique, RD128, \\ F-91127 Palaiseau Cedex, France \\ ${ }^{3}$ Centre de Photonique Biomédicale, Centre Laser de l'Université Paris-Sud, Univ Paris-Sud, Orsay, France \\ ${ }^{*}$ Corresponding author: sandrine.leveque-fort@u-psud.fr
}

Received 9 July 2007; revised 25 September 2007; accepted 27 September 2007; posted 10 October 2007 (Doc. ID 84996); published 19 November 2007

\begin{abstract}
Two-photon fluorescence microscopy is widely applied to biology and medicine to study both the structure and dynamic processes in living cells. The main issue is the slow acquisition rate due to the point scanning approach limiting the multimodal detection $(x, y, z, t)$. To extend the performances of this powerful technique, we present a time-resolved multifocal multiphoton microscope (MMM) based on laser amplitude splitting. An array of $8 \times 8$ foci is created on the sample that gives a direct insight of the fluorescence localization. Four-dimensional (4D) imaging is obtained by combining simultaneous foci scanning, timegated detection, and $z$ displacement. We illustrate time-resolved MMM capabilities for 4D imaging of a photosensitizer inside living colon cancer cells. The aim of this study is to have a better understanding of the photophysical processes implied in the photosensitizer reactivity. () 2007 Optical Society of America

OCIS codes: $180.2520,170.3650,180.4315,180.6900$.
\end{abstract}

\section{Introduction}

Fluorescence microscopy has become a powerful tool to probe cellular activity [1]. Combined with twophoton excitation [2], fluorescence measurements directly benefit from all the advantages associated with multiphoton excitation: inherent three-dimensional (3D) resolution without the use of a confocal pinhole, infrared lasers enhanced depth penetration, and photodamages or photobleaching are confined to the small focal volume. While 3D intensity images only reveal organization and/or compartmentalization of cell biomolecules, dynamic measurements such as fluorescence lifetime acquisition allow one to probe activity and interactions in a single living cell. As a consequence, for a complete and relevant study on biological samples the development of multimodal

0003-6935/07/338045-07\$15.00/0

(C) 2007 Optical Society of America microscopy, combining a number of fluorescence measurable parameters (intensity, emission spectrum, lifetime) and 3D imaging, has become essential and can provide novel insights into the biophysical properties of a sample with high molecular specificity and sensitivity.

The main drawback of conventional two-photon microscopy is intrinsic of its scanning mode, which can become rapidly time consuming. Consequently, to benefit from two-photon microscopy in a large range of biomedical fields, the imaging rate must be increased. By illuminating the sample with several excitation points, multifocal multiphoton microscopy (MMM) [3] reduces the acquisition time proportionally to the number of excitation beams used. Several approaches have already been developed to generate multiple foci and we can broadly classify them in two groups that differ in the way they divide the initial laser beam into beamlets bring either wavefront or amplitude division. Systems 
based on wavefront division already proposed can be a disk of a microlens [4,5], a microlens array $[6-8]$, or a diffractive optical element $[9,10]$; for the amplitude division approach the implementation of a cascade of beam splitters [11], or a beam splitter associated to multiple mirrors [12-14] have been presented. A complete review on these approaches can be found in [3]. Briefly, wavefront division on a microlens is typically obtained by expanding the laser beam on microlenses leading to a poor efficiency of light usage $(\sim 25 \%[3,6])$ and lower amplitude for outer beams. New microlens disk designs with higher filling factor and a good antireflection coating should permit higher transmittance. However it is usually associated with a spinning disk avoiding dead times. Regarding the amplitude division approach, all beamlets are a replica of the initial beam with a high transmission of incident light $(90 \%$ $[12,14])$ and a good uniformity between the beams can be obtained leading to a homogenously illuminated field of view. Furthermore the beam splitting induces different optical paths that provide an intrinsic minimization of the cross talk; the main drawback is related to the scanning technique limited to galvanometric mirrors or stage scanning involving dead times. The various MMM setups have been combined with different detection techniques $[5,14,15]$. We present here the development of a MMM based on a beam splitters approach, which allows us to acquire for the first time, to the best of our knowledge, both intensity and lifetime images at different $z$ positions. $3 \mathrm{D}$ lifetime imaging [four-dimensional (4D) imaging] using a wide field time-gated technique can be obtained with $25 \mathrm{ps}$ resolution and thus provides a drastic increase in contrast. We also demonstrate that the acquisition rate of our MMM permits highly photosensitive biological study such as 4D imaging of photosensitizer localization inside living cells.

\section{Multiple Foci Generation}

Our multifocal multiphoton microscopy system is derived from the work of Nielsen et al. [12], and we previously described the principle for generating a line of eight beams [14]. Although a reduced acquisition time was obtained, it did not allow to combine 3D acquisition using tunable excitation with fluorescence lifetime imaging (FLIM). To be able to address such performances, we developed a homemade system composed of two beam splitters creating an $8 \times 8$ array presented in Fig. 1. These two beam splitters are put in series, each composed of four high-reflectivity mirrors and a 50\% dichroic mirror (suprasil, $60 \mathrm{~mm} \times 40 \mathrm{~mm} \times 2 \mathrm{~mm}, P$ polarized, spectral bandwidth $750-850 \mathrm{~nm}$ ). The $50 \%$ mirrors have been designed to be as compatible as possible $(750-850 \mathrm{~nm})$ with the large tunability of the femtosecond Ti:sapphire laser. After a series of reflections and transmissions, a line of eight $P$-polarized beams is obtained. This line is turned upright using a periscope, each beam is split again into eight beams by a second beam splitter identical to the first one. A ma-

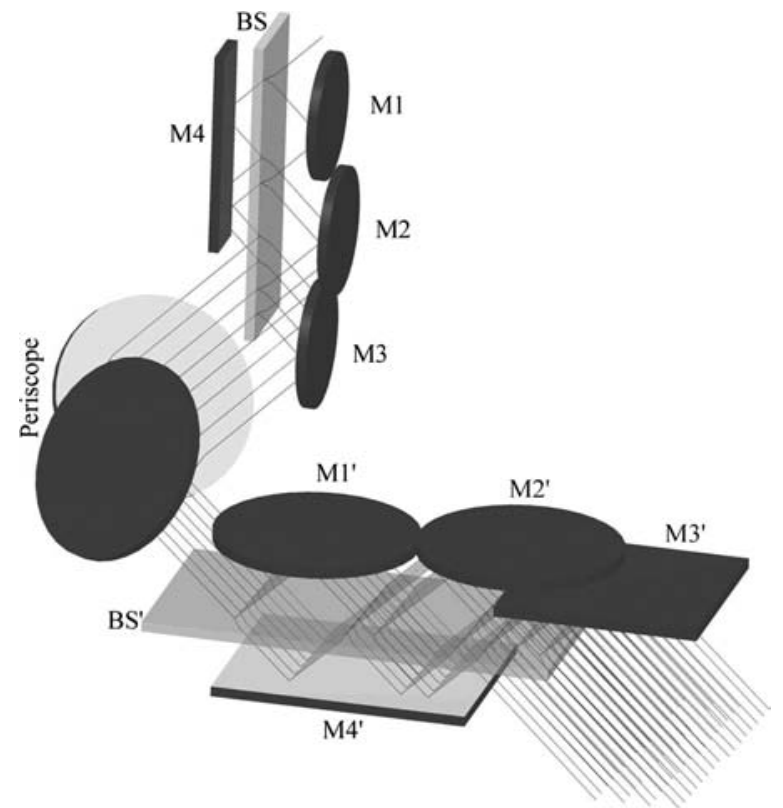

Fig. 1. Beam splitters principle: each beam splitter is composed of $50 \%$ dichroic mirror (BS, $\mathrm{BS}^{\prime}$ ) associated to four mirrors (M1-M4, M1'-M4'). The first system generates a line of eight points, rotated by using a periscope into a column, which entered the second beam splitter creating $8 \times 8$ excitation beams.

trix of 64 excitation beams is then generated. As the $50 \%$ mirror is $P$ polarized in both beam splitters (and the periscope turns the polarization) a $\lambda / 2$ wave plate is inserted between the first and second beam splitters (not represented in Fig. 1). This geometry leads to intrinsic different optical paths avoiding cross talks at the excitation. To be imaged separately in the focal plane of the objective, all the beams are slightly tilted using mirrors M1-M3 and M1'-M3', and their focus point is relayed onto the objective back-focal plane. For our applications, chosen angles lead to beam separation of $3 \mu \mathrm{m}$ in the $x$ direction and of $5 \mu \mathrm{m}$ in the $y$ direction. Those parameters can be easily adjusted by modifying the angles and/or changing distances between the mirrors and the 50\% dichroic mirror. We measured some small intensity inequalities not higher than $20 \%$ between two beams mainly due to the $50 \%$ dichroic mirror coating. Regarding pulse durations, autocorrelation measurements have been performed giving a mean value time width of $190 \mathrm{fs}$ for each beam. The maximum power available for each focus is $\sim 5 \mathrm{~mW}$ (measured at the objective back-focal plane). We applied this MMM to cell experiments and first established a major advantage of our setup. With the increase of the excitation beams number to 64 covering an $\sim 24 \mu \mathrm{m} \times 40 \mu \mathrm{m}$ area, it was possible to have a first insight of the fluorescence image of our sample, even without scanning the beams over the sample to reconstruct the entire image. For example, in the context of early bladder diagnosis using localization of EA 50 Papanicolaou staining [16], fixed foci [Fig. 2(a)] can reveal extracellular fluorescence; this localization was confirmed after foci scanning [Fig. 2(b)]. 

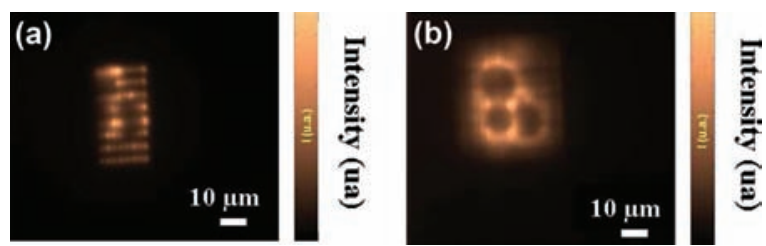

Fig. 2. (Color online) (a) Direct insight of the fluorescence localization with the 64 foci fixed on a cluster of three urothelial cells, (b) intensity image obtained after beams scanning for the same sample area as in (a).

Our setup also possesses a certain flexibility of use: indeed as the relative distance between two foci can be adjusted for given applications it can be used to probe only specific points inside the cells with a fast response and high signal-to-noise ratio. A second main advantage of the beam-distance adjustment is to choose it properly regarding scattering properties of the sample, and so avoid cross talk at the detection.

\section{Time-Gated Multifocal Multiphoton Microscopy}

\section{A. Experimental Set-Up}

Our time-resolved MMM is based on an Olympus IX 71 inverted microscope and the principle of the setup is presented in Fig. 3. The initial beam from a modelocked Ti:sapphire laser (100 fs, $800 \mathrm{~mW}, 76 \mathrm{MHz}$, $700-980 \mathrm{~nm}$, Mira from Coherent) is divided into 64 beams with the optical system described above. The tilting of the beams allows one to focus them on the $x-y$ galvanometric mirrors, whose scanning rate is typically from 50 to $1000 \mathrm{~Hz}$ depending on the fluorescence quantum yield of the sample.

The focusing point is relayed onto the back aperture of the objective (Zeiss, 63×, NA 1.4) using a telescope. The objective is mounted on a piezostage (Pifoc Polytech PI) for $z$ stacking. The fluorescence emitted by each excited point is imaged by a high-rate imager (HRI: $76 \mathrm{MHz}$, gate width from 200 to 1000 ps, delay 25 ps, Kentech Instruments Ltd.) optically coupled to a 12-bit CCD camera (Hamamatsu, model orca ER used in binning mode $2 \times 2$ ). A telescope can be inserted in front of the HRI depending on the required field of view and resolution. To reconstruct the intensity image of the studied sample, the signal on the CCD camera is integrated during the simultaneous scanning of the 64 foci using galvanometric mirrors, which gives directly the intensity image without any further treatment. The fluorescence lifetime is measured by sampling the fluorescence emitted following an excitation pulse, thus leading to a set of time-gated intensity images. Each pixel of the set is fitted with a specific algorithm (the LevenbergMarquardt nonlinear least-squares-fitting algorithm) and a typical area of the sample can be selected applying an appropriate threshold.

\section{B. Resolution}

The lateral resolution of the foci has been measured using a fluorescein layer for different detection configurations: either implementing a CCD camera for intensity imaging [typical image presented in Fig. 4 or using time-gated detection (CCD camera coupled

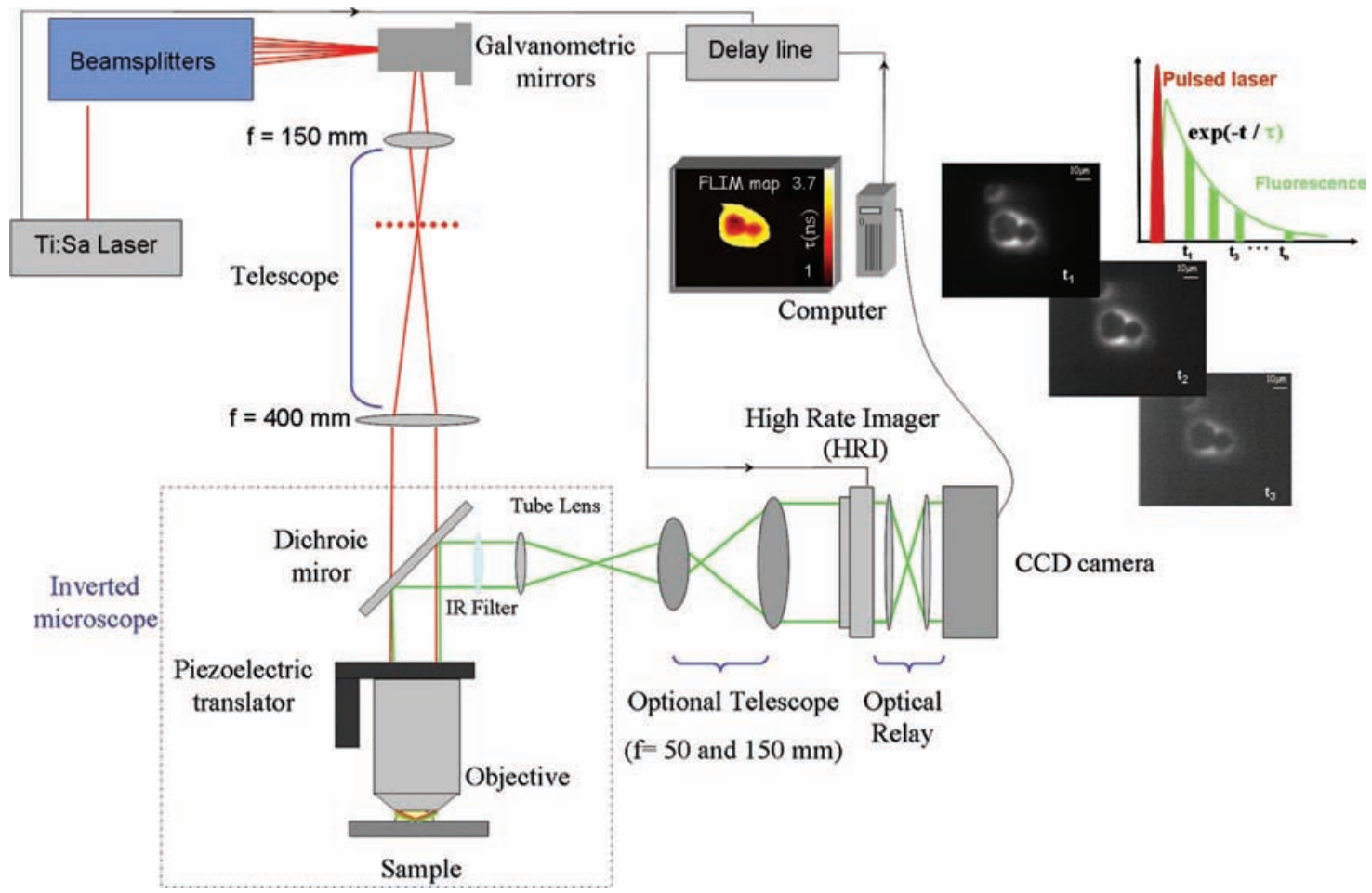

Fig. 3. (Color online) Schematic of the 3D time-resolved MMM and of the time-gated detection. In this case, $n$ time-gated images have been recorded at $t_{1}, t_{2}, t_{3}$, and $t_{n}$ after the excitation pulse. Consequently, we can thus reconstruct the lifetime map of the sample after fitting each pixel with a monoexponential. 


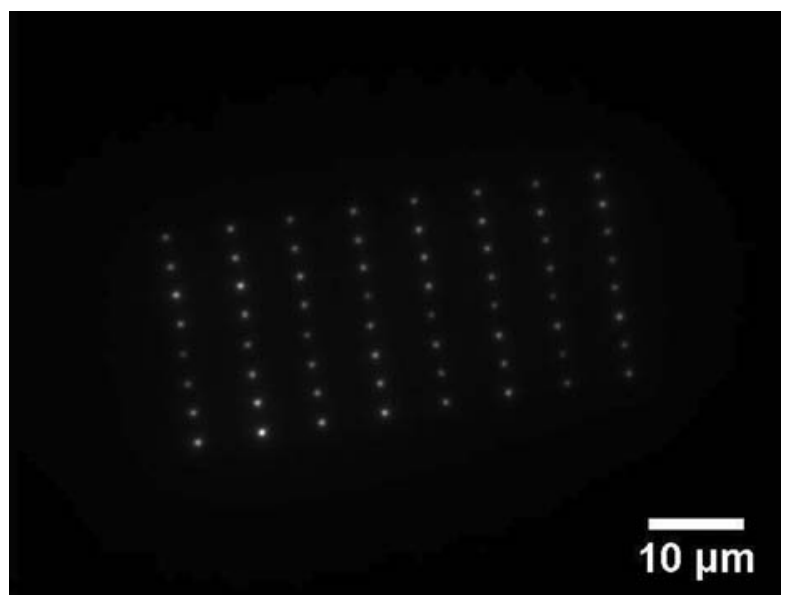

Fig. 4. Typical intensity image of the 64 foci on a fluorescein layer using $63 \times$ objective (NA 1.4) and only a CCD camera for detection (OrcaER Hamamatsu).

to HRI)]. For an excitation wavelength of $800 \mathrm{~nm}$ and a Zeiss $63 \times / 1.4$ immersion oil objective we measured FWHM of $600 \mathrm{~nm}$ using only the CCD camera at the detection (cf., Fig. 5). The diminished resolution in comparison to its theorical limit is the result of the slight underfilling of the back aperture to maximize excitation light throughput for all the beams.

Using the time-gated detection system, due to the low resolution of the HRI (up to $12 \mathrm{lppm}^{-1}$ ), the foci appear bigger (FWHM $1500 \mathrm{~nm}$ ). Depending on the resolution needed, the fluorescent image can be magnified before the HRI with a telescope (here typically a factor of 3, as it is detailed in Fig. 3) to prevent of resolution loss.

The axial resolution has been estimated by measuring the edge response for a $10 \mu \mathrm{m}$ diameter fluorescent bead deposited on the coverslip (Fluosphere, Molecular Probes). In the case of a simple Gaussian beam approximation [6], we use the following analytical formula to evaluate the approximate edge:

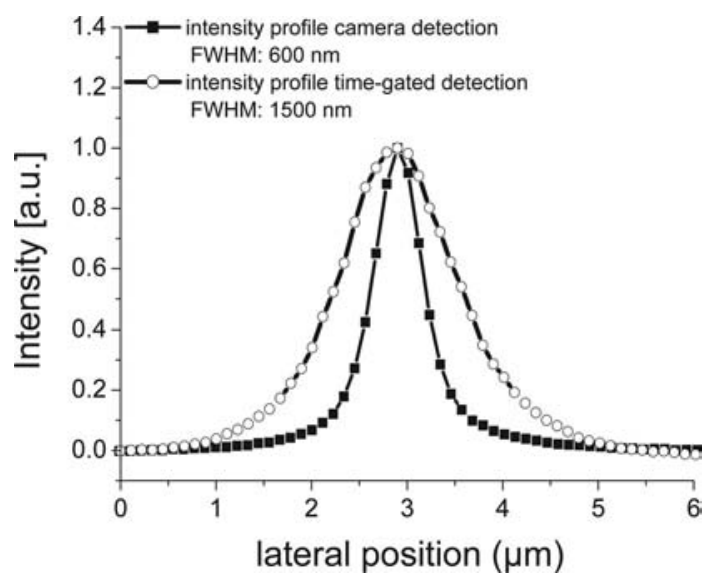

Fig. 5. Lateral resolution measured upon $800 \mathrm{~nm}$ excitation of a fluorescein layer $(63 \times$ objective, NA 1.4), using a CCD camera only or coupled to a high-rate imager (HRI) for time-gated detection.

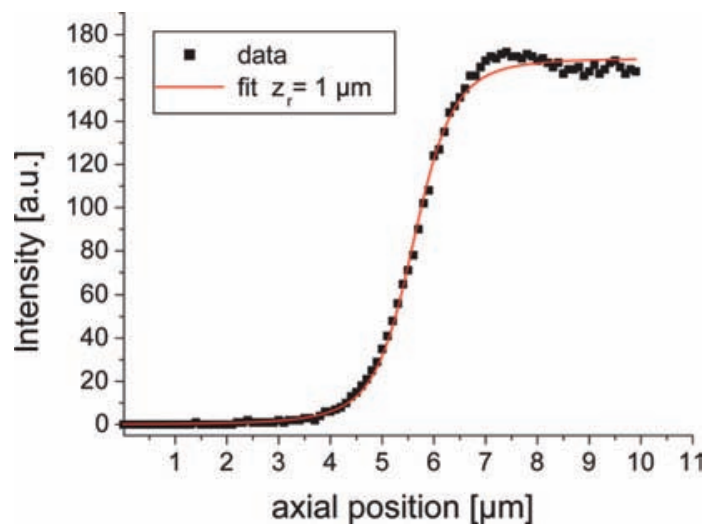

Fig. 6. (Color online) Axial response for a single beam on a $10 \mu \mathrm{m}$ diameter fluorescent bead, $z_{r}=1.0 \mu \mathrm{m}$.

$I_{\text {edge }}=\frac{A}{\pi}\left(\frac{\pi}{2}+\arctan \left(\frac{z-z_{0}}{z_{R}}\right)+\frac{1}{\frac{z_{R}}{z-z_{0}}+\frac{z-z_{0}}{z_{R}}}\right)$,

where $z_{R}$ is the Rayleigh length given by $z_{R}$ $=1.169\left(n \lambda / \mathrm{NA}^{2}\right), A$ is the intensity normalization factor, $z$ is the axial coordinate, $z_{0}$ is the position of the coverslip-fluorescent sample interface, NA is the numerical aperture of the objective lens, $n$ is the refractive index of the fluorescent sample, and $\lambda$ is the excitation wavelength (NA 1.4, $n=1.5, \lambda=$ $800 \mathrm{~nm}$ ). For a typical focus, the axial response is represented in Fig. 6, giving $1.0 \mu \mathrm{m}$ close to the theoretical expected value $0.7 \mu \mathrm{m}$. Small deviations from the model for $z>7 \mu \mathrm{m}$ are certainly due to refractive index mismatch $[6,17]$.

The accuracy of our time-resolved MMM system has been evaluated on various biological systems in comparison with a traditional two-photon microscope using time-correlated single-photon counting (TCSPC) $[16,18]$. In Fig. 7 , we represent the fluorescence decay for the photosensitizer pyropheophorbide-a methylester (PPME) in an ethanol solution by TCSPC and time-resolved MMM methods. We observe a good

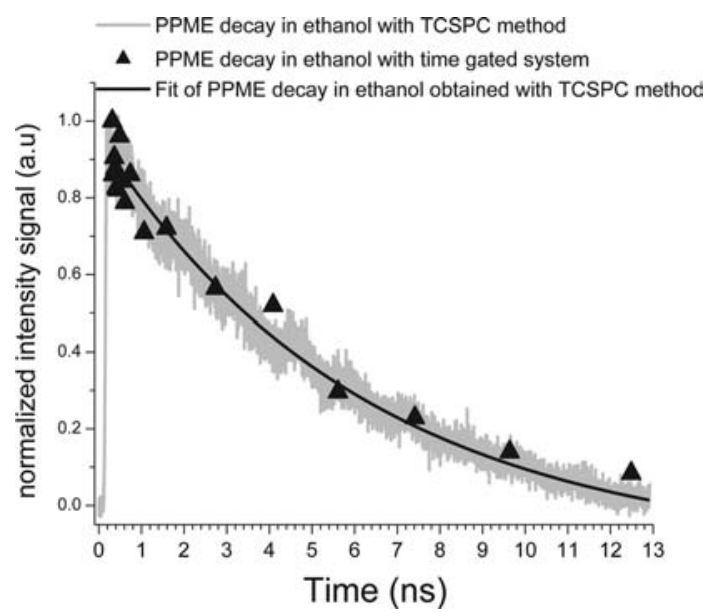

Fig. 7. Fluorescence decay of the photosensitizer PPME in an ethanol solution for TCSPC and time-gating methods. 

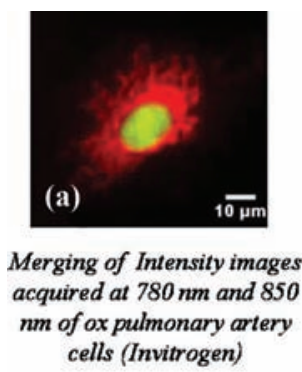

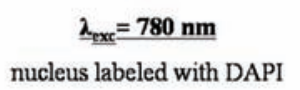

nucleus labeled with DAPI

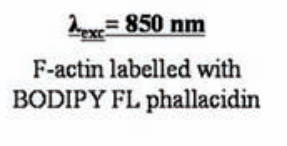

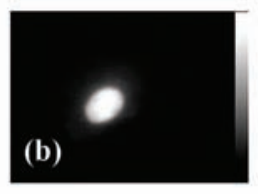

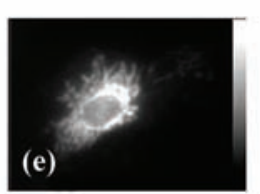

Intensity images
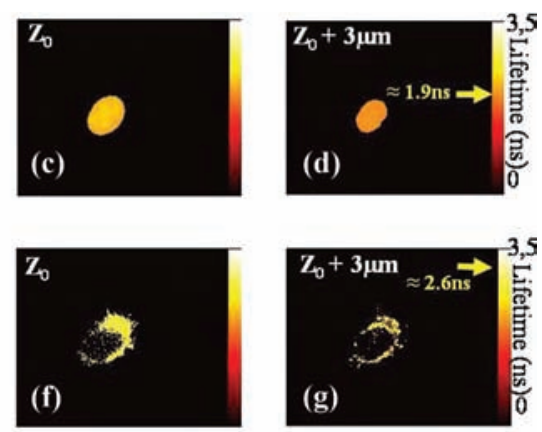

Lifetime images

Fig. 8. (Color online) Fluorescence image of multilabeled ox pulmonary artery endothelial cells. (a) Overlay of intensity image obtained with $780 \mathrm{~nm}$ excitation (in light gray, green online) and $850 \mathrm{~nm}$ excitation (in dark gray, red online). (b)-(d) Images under $780 \mathrm{~nm}$ excitation mainly efficient for nuclei labeling: (b) Intensity image; (c), (d) lifetime images for two different $z$ planes. (e)-(g) Images under $850 \mathrm{~nm}$ excitation mainly efficient for microtubule labeling: (e) intensity image; (f), (g) lifetime images for two different $z$ planes.

agreement with values of $6.2 \pm 0.1 \mathrm{~ns}$ for TCSPC and $5.9 \pm 0.4 \mathrm{~ns}$ for time-resolved MMM; the reduced acquisition time obtained in time-resolved MMM is at the cost of a slightly higher standard deviation.

\section{Tunability}

The large tunability of the Ti:sapphire laser allows a selective excitation of dye and so combines structural and functional information with a dynamic measurement of the fluorescence inside cells. This has been proved on microscope slides containing multilabeled cell preparations (Ox pulmonary artery endothelial cells): mitochondria are stained with Mito-Tracker Red CMXRos, F-actin are labeled with BODIPY FL phallacidin, and the nuclei with DAPI.

4D images are available for two excitation wavelengths (780 and $850 \mathrm{~nm}$ ) as partly depicted in Fig. 8. Fluorescence intensity images can be obtained at both excitation wavelengths and merge in false color as represented in Fig. 8(a). As depicted from Figs. $8(\mathrm{~b})-8(\mathrm{~d})$, the $780 \mathrm{~nm}$ excitation light performed essentially the excitation of nuclei labeling (DAPI) and a mean lifetime of $1.9 \pm 0.1 \mathrm{~ns}$ was obtained in the different $z$ planes. Whereas upon $850 \mathrm{~nm}$ excitation represented in Figs. 8(e)-8(g), microtubules labeling (BODIPY FL phallacidin) is mainly excited (and probably also the MitoTracker but very faintly) and the mean lifetime is around $2.6 \pm 0.3 \mathrm{~ns}$ for the different $z$ planes. Consequently, our system allows us to discriminate several components presenting specific lifetimes through selective excitation.

\section{Four-Dimensional Imaging of a Photosensitizer Inside Living Cells}

Our setup has revealed its real relevance for typical biological systems quite fragile or unstable due to their intrinsic nature or the presence of exogenous molecules. The most representative case is the study of a photosensitizer PPME, a promising molecule for photodynamic therapy (PDT). Indeed, it has been demonstrated that PPME produces less long-term skin photosensitivity than Photofrin [19]. Usually during PDT, the tumor is mainly destroyed by the radical oxygen species (ROS) that could be produced due to two different mechanisms in the presence of oxygen. The mechanism called type I results from the charge transfer reaction between the photoexcited drug and a biological substrate leading to ROS generation such as superoxide anion $\mathrm{O}_{2}^{-}$and hydroxyl radical $\mathrm{OH}^{\bullet}$. The type II mecnanism is a reaction between the photoexcited sensitizer in its triplet state and ${ }^{3} \mathrm{O}_{2}$ to form singlet oxygen ${ }^{1} \mathrm{O}_{2}$. It was previously reported $[19,20]$ that ${ }^{1} \mathrm{O}_{2}$ is involved in the necrosis of human colorectal carcinoma cell line (HCT 116) but that photoexcited PPME can also trigger apoptosis of tumoral cells mediated by $\mathrm{OH}^{\bullet}$ or $\mathrm{O}_{2}^{-}{ }^{-}$. This suggests that the singlet excited state of PPME can be involved in its photoreactivity. The purpose of this work is to evidence this mechanism by studying the fluorescence dynamic properties of PPME (SigmaAldrich) in HCT 116 cells. The absorption and fluorescence properties of the photosensitizer have already been well-characterized $[19,20]$ and the molecule presents a maximum fluorescence emission $\sim 680 \mathrm{~nm}$. Its properties as a photosensitizer require specific experimental conditions: thus the exposure time to laser beam of the HCT-116 cells in the presence of PPME must be drastically reduced to avoid cell photodestruction. Lifetime images were first obtained using a classic two-photon microscope coupled with the TCSPC system (acquisition $1 \mathrm{~s} /$ point). Only 20 points of lifetime measurements were possible before cell damages (Fig. 9), compromising the PPME photoreactivity study. (a)

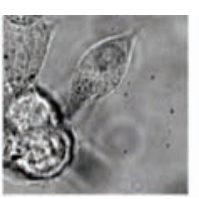

(b)

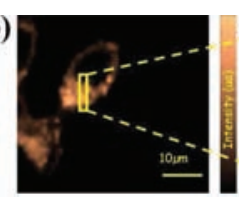

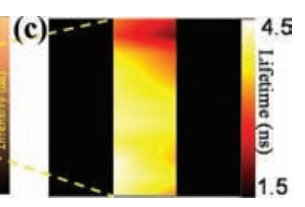

Fig. 9. (Color online) Results obtained with a classical two-photon microscope (excitation at $860 \mathrm{~nm}$ ) using TCSPC method as detection system on HCT 116 cells incubated with PPME ( $5 \mu \mathrm{M}$, overnight). (a) Transmittance image, (b) fluorescence intensity image $(50 \mu \mathrm{m} \times 50 \mu \mathrm{m})$, and (c) lifetime measurements $(2 \mu \mathrm{m} \times$ $10 \mu \mathrm{m}$, time exposure: $1 \mathrm{~s}$ per point). 
(a)

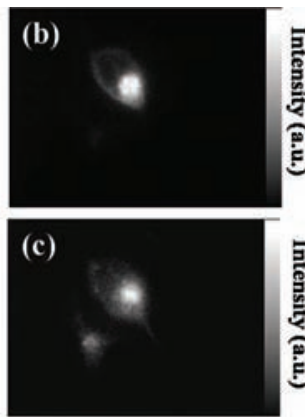

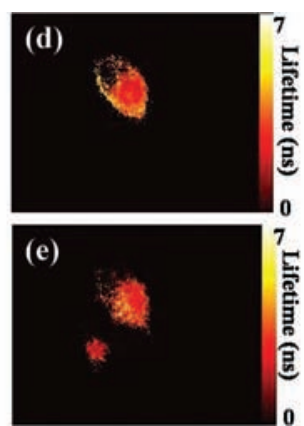

(f)

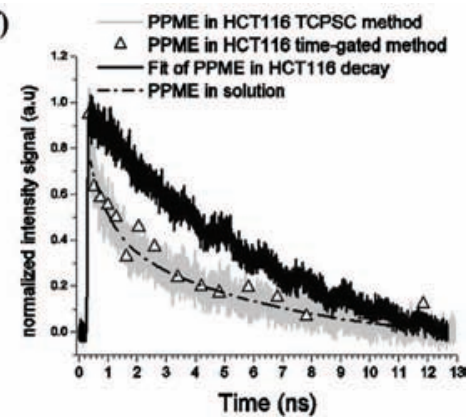

Fig. 10. (Color online) 4D imaging of PPME inside HCT 116 cells acquired with the MMM system: (a) transmittance image; (b), (c) fluorescence intensity images of HCT-116 cells after overnight incubation with PPME at $5 \mu \mathrm{M}$ at different $z$ positions extracted from a stack of 40 images (steps of $0.5 \mu \mathrm{m}$ ); (d), (e) lifetime image associated; (f) PPME decay in solution compared to cellular medium (HCT 116) for the two techniques.

The MMM approach can successfully address this issue. Beside the advantage of preserving biological samples, this method also gives the possibility of measuring both 3D intensity and lifetime images of PPME inside the entire cell. Figures 10(b) and 10(c) show intensity images of PPME location at different $z$ positions, preferentially in the cytoplasm. Intensity images have been obtained by scanning the galvanometric at $100 \mathrm{~Hz}$ for an area restricted to the cell $(44 \mu \mathrm{m} \times 60 \mu \mathrm{m})$ leading to $4 \mathrm{~s}$ of acquisition time. For lifetime measurements depending on the accuracy needed, the number of time-gated images can be adjusted to obtain either a simple contrast (two timegated images, FLIM map in $8 \mathrm{~s}$ ), or a decay sampling allowing more complex analysis (typically 16 timegated images, acquisition time $1 \mathrm{~min}$ ). The second approach has been followed here, and whatever the $z$ plane, lifetime maps are homogenous [Figs. 10(d) and 10(e)]. Regarding lifetime results in detail, both the TCSPC and time-gated systems give an average lifetime of $3.6 \pm 0.3 \mathrm{~ns}$ for PPME inside cells [see Fig. $10(f)]$ revealing a significant quenching of its fluorescence in cellular medium in comparison to lifetime measured in solution. Such a process is in good agreement with a charge transfer reaction from PPME in its singlet excited state with its environment leading to formation of $\mathrm{ROS}_{2}^{-\bullet}$ and $\mathrm{OH}^{\bullet}$.

\section{Conclusion}

4D imaging of the photosensitizer clearly proves the efficiency, the real pertinence, and all the advantages of using our homemade system for living cells study. We succeeded in acquiring 3D time-resolved images for a colorectal carcinoma cell line incubated with a photosensitizer (PPME) while limiting sample photodestruction. We are still optimizing our setup, especially in terms of signal-to-noise ratio, and different approaches are tested for quicker lifetime measurements and fitting (e.g., rapid lifetime determination algorithm [21,22]). Cell imaging has demonstrated the high potential of the time-resolved MMM. Tissue imaging could also take advantage of this approach. While two-photon excitation increases depth penetration, tissue imaging still suffers from scattering and cross talks at the detection; we could then benefit from interbeam distance tunability to reduce this major issue.

We gratefully thank Lisiane Delanaye, of the Biomedical Spectroscopy Laboratory in the University of Liège, who gave us the opportunity of working on her samples (human colorectal carcinoma cell line, HCT-116, and PPME). This work has been partially funded by the research program ACI NanoscienceNanotechnologie from the CNRS and by a Contrat Plan Etat Region (POLA 2000-2006) from French State and Conseil Général de l'Essonne.

\section{References}

1. R. Yuste, "Fluorescence microscopy today," Nat. Methods 2, 902-904 (2005).

2. W. Webb, "Nonlinear magic: multiphoton microscopy in the biosciences," Nat. Biotechnol. 21, 1369-1377 (2003).

3. J. Bewersdorf, A. Egner, and S. W. Hell, Handbook of Biological Confocal Microscopy, J. Pawley, ed. (Springer, 2006), pp. 550-560.

4. J. Bewersdorf, R. Pick, and S. H. Hell, "Multifocal multiphoton microscopy," Opt. Lett. 23, 655-657 (1998).

5. M. Straub and S. H. Hell, "Fluorescence lifetime threedimensional microscopy with picosecond precision using a multifocal multiphoton microscope," Appl. Phys. Lett. 73, 1769-1771 (1998).

6. A. H. Buist, M. Muller, J. Squier, and G. J. Brakenhoff, "Real time two-photon absorption microscopy using multipoint excitation," J. Microsc. 192, 217-226 (1998).

7. K. Fujita, O. Nakamura, T. Kaneko, M. Oyamada, S. Kawata, and T. Takamatsu, "Confocal multipoint multiphoton excitation microscope with microlens and pinhole arrays," Opt. Commun. 174, 7-12 (2000).

8. K. Fujita, O. Nakamura, T. Kaneko, M. Oyamada, T. Takamatsu, and S. Kawata, "Real-time imaging of two-photoninduced fluorescence with a microlens-array scanner and a regenerative amplifier," J. Microsc. 194, 528-531 (2000).

9. L. Sacconi, E. Froner, R. Antolini, M. R. Taghizadeh, A. Choudhury, and F. S. Pavone, "Multiphoton multifocal microscopy exploiting a diffractive optical element," Opt. Lett. 28, 1918-1920 (2003).

10. J. E. Jureller, H. Y. Kim, and N. F. Scherer, "Stochastic scanning multiphoton multifocal microscopy," Opt. Express 14, 3406-3414 (2006).

11. D. Fittinghoff, P. Wiseman, and J. Squier, "Wide-field multiphoton and temporally decorrelated multifocal multiphoton microscopy," Opt. Express 7, 273-279 (2000). 
12. T. Nielsen, M. Fricke, D. Hellweg, and P. Andresen, "High efficiency beam splitter for multifocal multiphoton microscopy," J. Microsc. 201, 368-376 (2001).

13. M. Fricke and T. Nielsen, "Two-dimensional imaging scanning by multifocal multiphoton microscopy," Appl. Opt. 44, 29842988 (2005).

14. S. Lévêque-Fort, M. P. Fontaine-Aupart, G. Roger, and P. Georges, "Fluorescence-lifetime imaging with a multifocal twophoton microscope," Opt. Lett. 29, 2884-2886 (2004).

15. L. Liu, J. Qu, Z. Lin, L. Wang, Z. Fu, B. Guo, and H. Niu, "Simultaneous time- and spectrum-resolved multifocal multiphoton microscopy,” Appl. Phys. B 84, 379-383 (2006).

16. K. Steenkeste, S. Lécart, A. Deniset, P. Pernot, P. Eschwège, S. Ferlicot, S. Lévêque-Fort, R. Briandet, and M. P. FontaineAupart, "Ex vivo fluorescence imaging of normal and malignant urothelial cells to enhance early diagnosis," Photochem. Photobiol. 83, 1157-1166 (2007).

17. S. Hell, G. Reiner, C. Cremer, and E. H. K. Stelzer, "Aberrations in confocal fluorescence microscopy induced by mismatches in refractive index," J. Microsc. 169, 391-405 (1993).

18. A. Deniset, S. Lévêque-Fort, M. P. Fontaine-Aupart, G. Roger, and P. Georges, "Multifocal multiphoton fluorescence lifetime microscopy for biomedical applications," Proc. SPIE 5860, 59-66 (2005).

19. J. Y. Matroule, C. M. Carthy, D. J. Granville, O. Jolois, D. W. Hunt, and J. Piette, "Mechanism of colon cancer cell apoptosis mediated by pyropheophorbide-a methylester photosensitization," Oncogene 20, 4070-4084 (2001).

20. L. Delanaye, M. A. Bahri, F. Tfibel, M. P. Fontaine-Aupart, A. Mouithys-Mickalad, B. Heine, J. Piette, and M. Hoebecke, "Physical and chemical properties of pyropheophorbide-a methylester in ethanol, phosphate buffer and aqueous dispersion of small unilamellar dimyristoyl-L- $\alpha$-phosphatidylcholine vesicles," Photochem. Photobiol. Sci. 5, 317-325 (2006).

21. S. P. Chan, Z. J. Fuller, J. N. Demas, and B. A. DeGraff, "Optimized gating scheme for rapid lifetime determinations of single-exponential luminescence lifetimes," Anal. Chem. 73, $4486-4490$ (2001).

22. J. Requejo-Isidro, J. McGinty, I. Munro, D. S. Elson, N. P. Galletly, M. J. Lever, M. A. A. Neil, G. W. H. Stamp, P. M. W. French, P. A. Kellett, J. D. Hares, and A. K. L. DymokeBradshaw, "High-speed wide-field time-gated endoscopic fluorescence-lifetime imaging," Opt. Lett. 29, 2249-2251 (2004). 